\title{
Data Acquisition System Prototype of the ITER Diagnostic Divertor Neutron Flux Monitor Testing at Research Nuclear Facilities
}

\author{
E.S. Martazov ${ }^{1}$, Yu.A. Paryshkin ${ }^{1}$, N.A. Selyaev ${ }^{1}$, V.A. Fedorov ${ }^{1}$, V.A. Vorobev $^{2}$, A.S. Dzhurik ${ }^{2}$, \\ Yu.A. Kashchuk ${ }^{2}$, S.Yu. Obudovsky ${ }^{2}$, and M.V. Bulavin ${ }^{2,3}$ \\ ${ }^{1}$ National Research Nuclear University "MEPhI", Russian Federation \\ ${ }^{2}$ Institution "Project Center ITER", Russian Federation \\ ${ }^{3}$ Joint Institute for Nuclear Research, Russian Federation \\ ESMartazov@mephi.ru
}

\begin{abstract}
The Divertor nuclear flux monitor (DNFM) is one of the ITER neutron diagnostics. This diagnostic consists of the three same subsystems. Each subsystem concludes the detector module with fission chambers (FCs) and the data acquisition system (DAQ). To solve the task of the neutron flux measurements in the range of 7 orders of magnitude and $1 \mathrm{~ms}$ of time resolution the multidetector module is used. To confirm the possibility of the neutron flux measurements in a wide range using such a detector module and to evaluate the characteristics of the DAQ prototype a number of tests were carried out under conditions of the intense neutron radiation. The detector module and the DAQ, which are the prototype of the equipment planned for use on site were used for the tests. The tests were carried out at the plasma neutron diagnostic stand based on the NG-24M neutron generator and at the IBR-2 pulsed reactor of the Joint Institute for Nuclear Research. During the tests at the plasma neutron diagnostic stand the data for the calibration of the DNFM DAQ measuring channels were collected. During the tests at the IBR-2 pulsed reactor the signals from the measuring channels of the DNFM subsystem were obtained while the neutron flux was changed. This report shows the test results and the subsystem calibration techniques.
\end{abstract}

Keywords -Fusion reactors, Tokamak devices, Neutrons, Reactor instrumentation.

\section{INTRODUCTION}

$\mathrm{T}$ HE neutron diagnostic "Divertor neutron flux monitor" (DNFM) is one of the instruments to determine the neutron yield and the thermonuclear power of the International thermonuclear experimental reactor ITER. According to the project requirements this diagnostic should provide the measurements of these parameters in the range of 7 orders of magnitude and $1 \mathrm{~ms}$ time resolution.

The DNFM diagnostic consists of the three identical subsystems. Each subsystem comprises the detector module and the data acquisition system (DAQ). The detector modules will be placed on the inner part of the ITER vacuum vessel under the divertor cassette. The expected neutron flux at the detector modules locations is from $10^{6}$ to $10^{13} \mathrm{n} \times \mathrm{cm}^{-2} \times \mathrm{s}^{-1}$. The neutron flux measurements should be provided with $1 \mathrm{~ms}$ of time resolution and $10 \%$ of uncertainty. To meet these requirements the multidetector module consists of six FCs with different sensitivities and the DAQ system processed FCs signals with different methods is used.

At the first stage the DAQ system prototype was manufactured. With this prototype the software for measuring and diagnostic procedures was checked and validated [1].

The next stage of the DNFM neutron diagnostic research and developing was field testing of the DAQ system prototype with the detector module mockup. The first aim of tests was to check the operation parameters of the DNFM subsystem under the conditions of neutron and gamma radiation. The second aim was to obtain the data to validate the methods and techniques of the calibration and combination of the measurement chains of each measurement channel taking into account the FC parameters.

\section{STRUCTURE OF THE DNFM SUBSYSTEM}

Figure 1 shows the structure of the DNFM subsystem for the single detector module. The detector module concludes two detector units (DU). Each DU is the combination of three FCs. DU U5 consists of $235 \mathrm{U}$ FCs and DU U8 consists of 238U FCs. The expected sensitivity of the FCs in the single DU has a ratio 1:10:100. Using such a detector module allows one to provide the dynamic range of the DNFM diagnostic.

The DNFM data acquisition system has a spatially distributed structure. The Preamplifier Unit (PAU) consists of six Preliminary amplifier modules (PAM) and the module rack. The main function of PAM is to separate the pulse and DC components of the FC signal and amplify the pulse signal. To reduce the communication lines length from the FCs and the influence of electromagnetic interference PAU will be placed in a shielded protective box in the Port Cell as close as possible to the detector module. The circuitry of the PAM modules has been optimized to minimize the effect of electromagnetic fields and radiation and to reduce the shielded volume.

The DNFM Signal Processing Unit (SPU) conclude six Control and preprocessing modules (CPPM) and six remote 


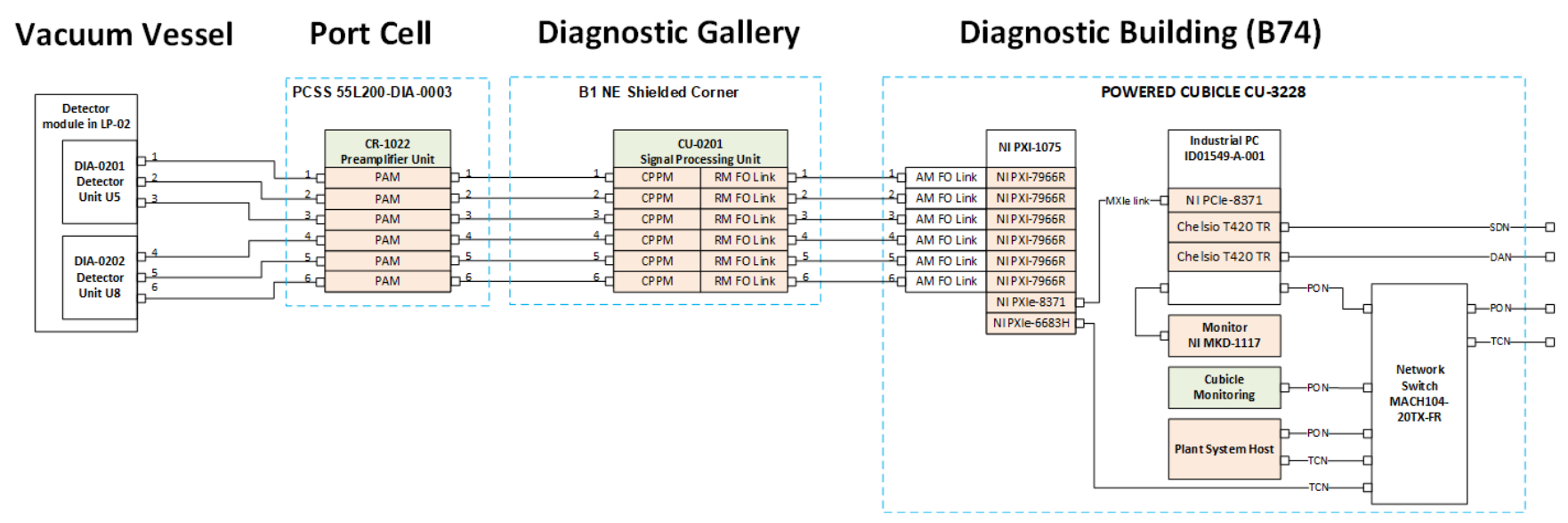

Fig. 1. The structure of the DNFM subsystem for single detector module.

fiber optic modules (RM FO). SPU will be placed in the gallery of the Tokamak building and will be connected to the PAU via communication lines of at least $30 \mathrm{~m}$ of length. The main functions of the SPU are the PAM signals conditioning and preliminary analog processing, converting it to digital form and transfering to the PXIe-system. The conversion of signals into digital form is carried out using an analog to digital convertor (ADC) with 14 bits resolution and $120 \mathrm{MS} / \mathrm{s}$ sample rate. The data from the ADC outputs are converted from parallel to serial form and transferred to the PXIe-system at $2.5 \mathrm{~Gb} / \mathrm{s}$ ratio. The modular design of the PAU and SPU allows, if necessary, to rapid replace the modules without dismantling the rest of the equipment.

The PXIe-system receives digital data from the SPU, processes them via the NI PXIe-7966R module software, and transfers the results for further processing and calculations to the industrial personal computer (PC). The industrial PC publishes "raw" and calculated data on the ITER plant-wide network. Herein and after the "raw" data means signals from the output of the analog part of the measuring chains converted into a digital code with a maximum sampling rate without any digital processing. The Industrial PC and PXIesystem will be placed in the diagnostic cubicle along with additional equipment in the diagnostic building.

Signals from the detector module to the BPU unit and from the BPU to the SPU unit are transmitted via triaxial cables. The SPU unit is connected to the PXI system via optical lines.

\section{COMBINATION OF THE DATA OF THE DNFM SUBSYSTEM MEASUREMENT CHAINS}

To increase the dynamic range of the neutron flux measurements using one FC the data acquisition system processes the signal from each chamber with four methods: pulse counting, fluctuation (MSV, Campbelling), current, and pulse-fluctuation. The pulse-fluctuation chain overlaps the operating range of the pulse counting and MSV chains. It had been included in the system processing channel to increase interference immunity and implement the principle of diversity. The main feature of this chain is the use of a charge- sensitive amplifier with a linear selector [2]. The selector is implemented in digital form in the FPGA software. The subsequent processing is provided by the PC software.

To take to a single physical value dissimilar data of all measuring chains of single FC channel in the areas of their combinations the system uses the concept of a "normalized" pulse count rate. For a pulse counting chain the "normalized" count rate is the count rate of impulses at a conditionally zero threshold, i.e. the number of all neutrons detected by the FC for a specified time frame. To calculate the "normalized" count rate of pulse counting chain the measured count rate should be multiplied by a dimensionless calibration factor (greater than one). Calibration factor depends on the magnitude of the threshold in the chain. For the rest chains it is also necessary to determine these coefficients (have the appropriate dimensions). For example for the current chain the calibration factor is inversely proportional to the average charge of the FC current pulse. To obtain the values of the neutron flux one should divide the "normalized" pulse count rates for each measuring chain by the pulse sensitivity coefficient of the FC. Ideally, the obtained neutron flux should be the same for all chains of all channels with the FC used in the DNFM diagnostic.

The following methods can be used to determine the calibration coefficients in the measuring chains of single channel of DAQ with the FC:

- Experimental method;

- Computational method;

- Mixed experimental and computational method.

\section{A. Experimental method}

The experimental method is the best in terms of the accuracy of determining the calibration coefficients. The method consists in exposing the FC to the sufficient neutron radiation to measuring the signals of the chains in the regions of their combination. The use of powerful neutron sources such as research reactors leads to high activation of the detector module and the impossibility of its further use as a result. Therefore, this method can be used at the ITER only after the first launches or during tests as a reference method for assessing the uncertainty of the remaining two methods. 


\section{B. Computational method}

The computational method requires mathematical modeling of both physical processes in the FC taking into account the characteristics of the electrodes, gas pressure, tracks of fission fragments, and the characteristics of the analog part and processing algorithms of the $\mathrm{I} \& \mathrm{C}$ digital parts. Calculations of FCs processes are complex and the results have significant errors.

\section{Mixed experimental and computational method}

The mixed experimental and computational method is based on the functions of the averaged pulse shape and the pulseheight spectrum, as well as the distribution of pulses by electric charges. These data are obtained experimentally for each FC. The pulse shape and pulse-height spectrum are recorded from the outputs of the pulse counting chains. The distribution of pulses by electric charges is recorded from the outputs of the pulse-fluctuation chains. The DAQ system implements the function of recording impulses (sampling frequency of signals $120 \mathrm{MS} / \mathrm{s}$ ) and spectra (up to 4096 channels). These data are the basic for subsequent mathematical or semi-natural modeling in order to calculate the calibration coefficients.

In mathematical modeling it is possible to use softwareimplemented FC models, in which the corresponding normalized pulse shape and pulse-height spectrum are set, and the models of the measuring chains signal responses.

In semi-natural modeling it is advisable to use a full-scale sample of a data acquisition system (a prototype, for example) and an I\&C complex (fission chamber signal simulator $[3,4]$ ), which implements the mathematical model of forming the FC signal with set parameters and provides an output of an electric pulse-current signal.

The last mentioned method of determining the calibration coefficients was verified during the tests.

\section{DNFM PROTOTYPE TESTING AT THE NEUTRON STAND}

The DNFM DAQ prototype tests with mockups of detector units were carried out at the neutron diagnostics stand of plasma of the JSC "SSC RF TRINITY". The stand is build on the basis of the NG-24M neutron generator [5]. Figure 2 shows photos of the equipment during tests at the neutron diagnostic stand of plasma.

During tests at the neutron stand the average pulse shapes and pulse-height spectra were recorded for six FCs of the detector module prototype. The data for calibrating the DAQ measuring chains were obtained and the relative sensitivity of the FCs of the detector units was estimated.

Some experiments were carried out to confirm the possibility of calculating the calibration coefficients by the semi-natural modeling method. To determine the characteristics the high sensitivity FC with a $235 \mathrm{U}$ was used. To increase the thermal neutron flux the FC was placed in the paraffin neutron moderator. This made it possible to obtain a counting rate of up to $5 \times 10^{6} \mathrm{~s}^{-1}$. The averaged over 1000 counts pulse shape and pulse-height spectrum were recorded. The average electrical charge per pulse has been calculated. The obtained data were set to the FC signals simulator built on the basis of NI equipment (NI PXIe-5451 generator and NI PXIe-7966R FPGA module for FlexRIO). To restore the current pulse shape which should have been set from the FC signal simulator to the PAM input the obtained pulse shape was processed using a dynamic model corresponding to the inverse transfer function of the pulse chain analog part. This allows one to simulate the FC output signal taking into account its parameters and characteristics of the communication line between the FC and the input of the preamplifier. The pulse-height spectrum was also normalized to set into the FC signal simulator. Figures 3 and 4 show the normalized averaged pulse shape and the pulse-height spectrum. They were set to the FC signal simulator during calibration of the measuring chains.

Figure 4 also shows the threshold set in the pulse counting chain and reflects the area with the total number of pulses that were registered in the chain (above the threshold) and the area of unregistered pulses (below the threshold level). For the current distribution the amount of registered pulses was $83 \%$ and unregistered pulses $-17 \%$.

Using the FC signal simulator the signals with count rate in the range from $1 \mathrm{E} 3$ to $1 \mathrm{E} 8 \mathrm{1} / \mathrm{s}$ were sequentially set to the input of the measurement channel of the DAQ prototype. The measuring chains data were recorded. Using the obtained data the calibration coefficients of the measuring chains were

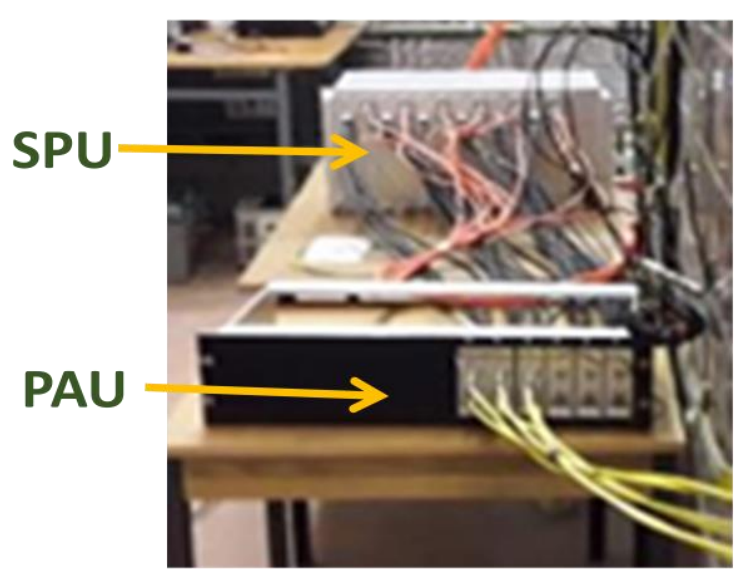

\section{Detector Module} mockup

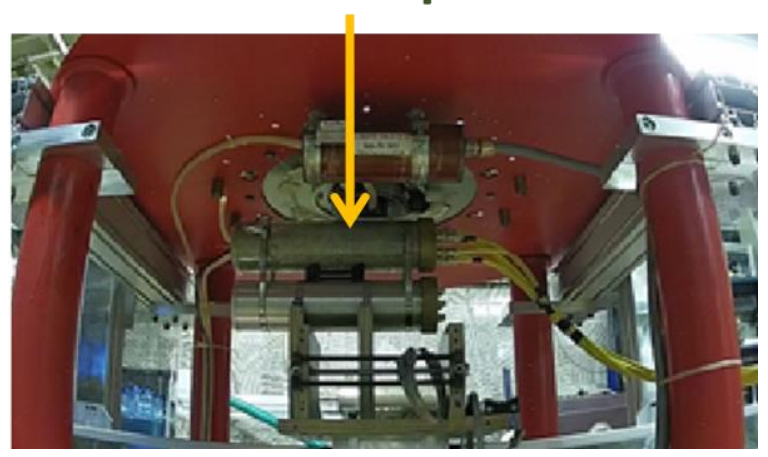

Fig. 2. Images of the equipment during tests at the plasma neutron diagnostics stand. 
calculated. The calibration coefficients were set into the prototype configuration file. Further, the data of the normalized count rate of the measuring chains were recorded during the process of changing of the neutron generator power. Figure 5 shows the measuring data of the normalized count rates of the DAQ measuring chains.

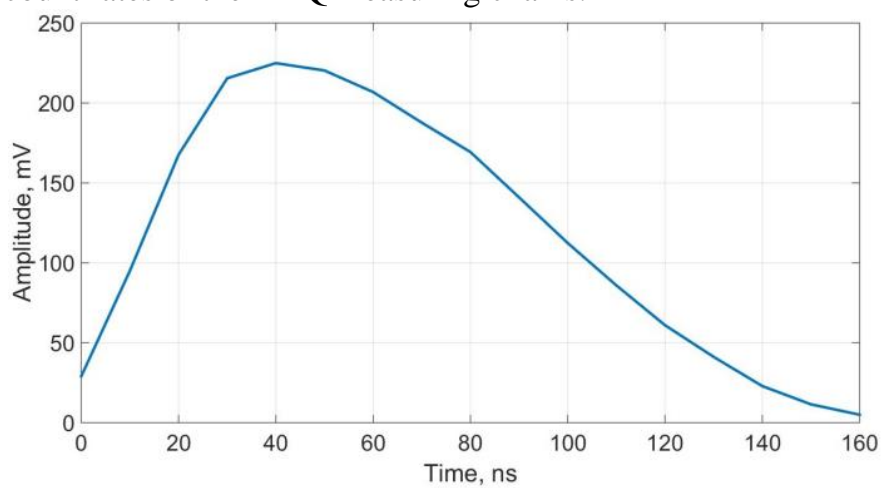

Fig. 3. Normalized averaged pulse shape of the high sensitivity FC with a $235 \mathrm{U}$ to set into the FC signal simulator.

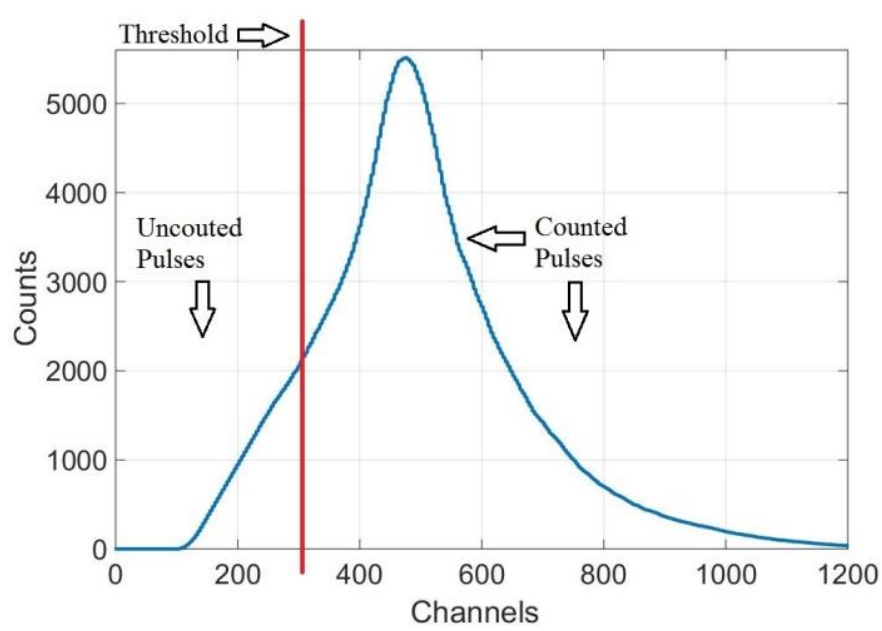

Fig.4 . Normalized pulse-height spectrum of the high sensitivity FC with a $235 \mathrm{U}$ to set into the FC signal simulator.

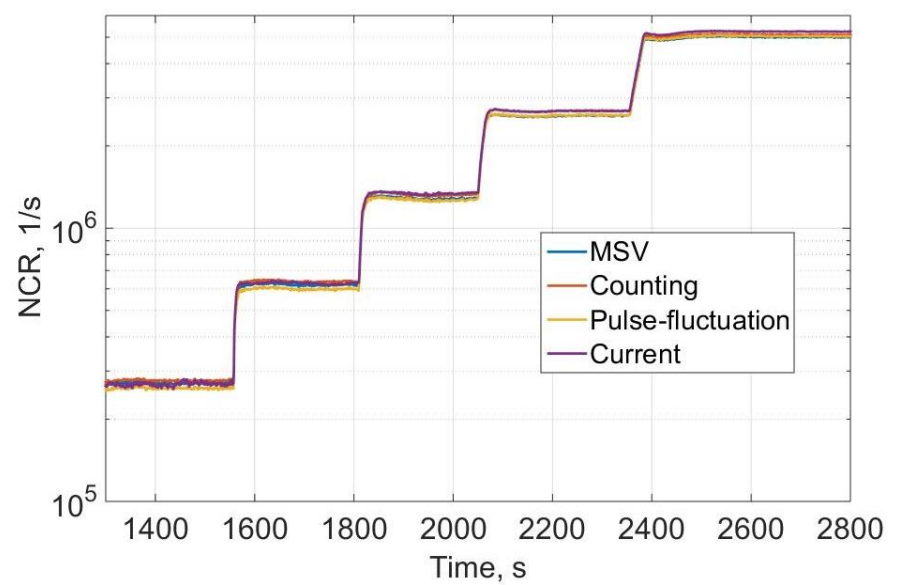

Fig. 5. Normalized count rates of the DAQ measuring chains in the tested range.

The deviations in the measuring data of the normalized count rate from the average value of all four measuring chains in the tested range did not exceed $2 \%$. This result shows the possibility of determining the calibration coefficients set of the
DAQ for calculating the normalized count rates in the chains by the mixed experimental and computational method.

\section{DNFM PROTOTYPE TESTING AT THE IBR-2M PULSED REACTOR}

The tests with the FC under conditions of intense neutron and gamma radiation were carried out at the IBR-2M pulsed reactor at JINR [6, 7]. During the tests the signals of the DAQ measuring chains with connected detector unit mockups were obtained at constant power levels and in the process of its change (ramp up and ramp down) of the reactor facility. A specific feature of the IBR-2M pulsed reactor is the repetitive power increase pulses with a frequency of $5 \mathrm{~Hz}$. This means that every $200 \mathrm{~ms}$ the neutron flux increases by several orders of magnitude in a time of the order of $240 \mu \mathrm{s}$. This feature of the reactor makes it possible to investigate the time resolution of the measurement channels and study the operation of the DAQ during short-term overloads of the measuring chains. But this had to be taken into account when processing the results. Figure 6 shows the experimental channel of the IBR-2 reactor where the detector module mockup was placed. Figures 7 and 8 show the normalized count rate of the MSV chain while the reactor power rump-up and at a constant power of the plant of $450 \mathrm{~kW}$ over a short time interval.

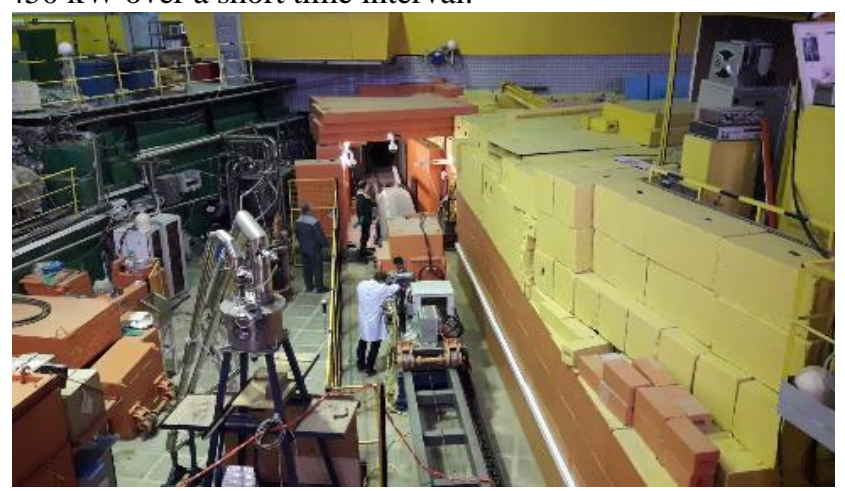

Fig. 6. Experimental channel of the IBR-2 reactor, where the detector module mockup was placed during the tests.

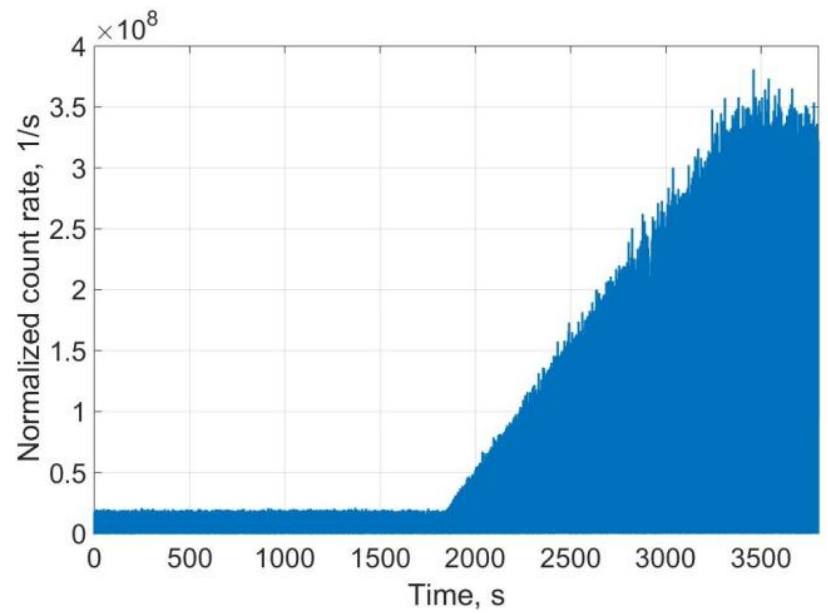

Fig. 7. Normalized count rate of the MSV chain while the reactor power rump-up 


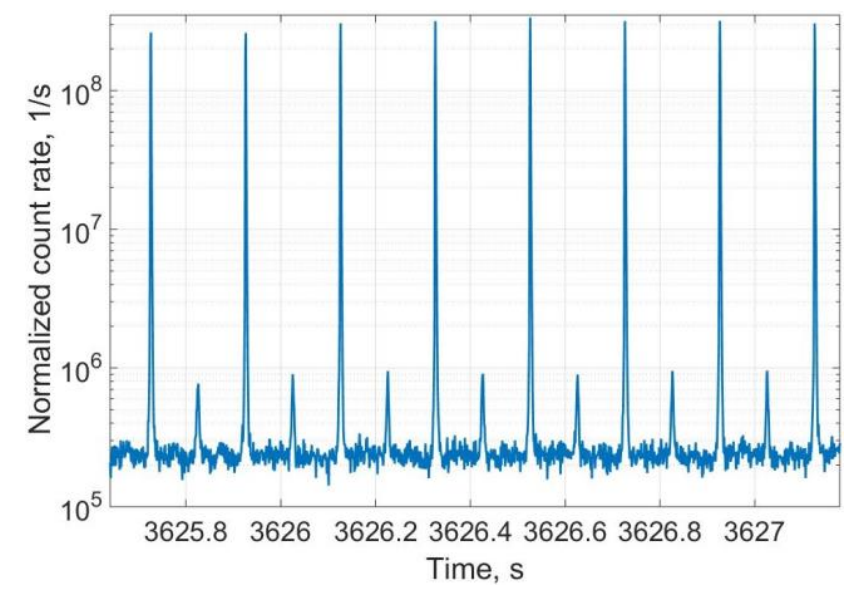

Fig. 8. Normalized count rate of the MSV chain at a constant power of the plant of $450 \mathrm{~kW}$ over a short time interval.

Despite the pulsed operation of the reactor, during the tests it was possible to obtain the averaged pulse shapes and pulseheight spectra for the four most sensitive to the neutron flux FCs and to estimate the boundaries of the linear operation regions of the measuring chains.

\section{CONCLUSIONS}

The tests of the DNFM data acquisition system prototype at research nuclear facilities within the FC multidetector units and communication lines, similar to those that are supposed to be used in the diagnostics "Divertor neutron flux monitor", confirmed the possibility of measuring the neutron flux in a wide range with specified measurements uncertainty and dynamic characteristics of measurement channels. During the tests methods of measurements and data processing were checked out.

An important result of the tests is the approbation of the mixed computational and experimental method for calibrating the measuring chains of the DAQ according to the data on the average pulse shape and the pulse-height spectra. The method makes it possible to determine the values of the calibration coefficients of the measuring chains during the in-situ calibration of the DNFM diagnostics using the neutron source at the ITER facility for subsequent set it into the DAQ configuration file. In case of a change in the pulse chains of the thresholds values (determined by noise, electromagnetic interference and the expected level of gamma) their calibration coefficients are easily corrected according to the pulse-height spectra. To automate this process, a service program is used that contains a database of spectra of all 18 fission chambers included in the DNFM diagnostics.

\section{ACKNOWLEDGMENT}

The authors would like to acknowledgment to the staff of the neutron diagnostics stand of plasma of the JSC "SSC RF TRINITY" and the IBR-2M pulsed reactor at JINR for their help in preparing and conducting the tests.

This work was carried out with the financial support of the
Institution "Project Center ITER" in the framework of the state contract with State Corporation "ROSATOM".

\section{REFERENCES}

[1] V.A. Fedorov, et al "Study of the Data Acquisition System for ITER Divertor Neutron Flux Monitor Diagnostic," IEEE Transactions on Nuclear Science, vol. 67, no. 4, pp. 688-693, April 2020.

[2] V.P.Alferov, et al "Applicaton of Modern Tools for NPP Neutron Monitoring Systems Upgrading," in Proc. International Topical Meeting on Nuclear Plant Instrumentation, Controls, and Human-Machine Interface Technologies (NPIC\&HMIT 2000), Washington, D.C., USA, November 2000, pp. 393-401.

[3] A.V. Batyunin, et al "Fission Chamber Simulator for Data Acquisition Performance Tests," in Proc. Conference on Advancements in Nuclear Instrumentation, Measurements Methods and their Application ANIMMA-2013, Marceille, France, June 2013, pp. 1-4.

[4] I. V. Alyaev, et al "Wide-range fission chambers signal simulator," in Proc. 10th International Topical Meeting on Nuclear Plant Instrumentation, Control, and Human-Machine Interface Technologies (NPIC\&HMIT 2017), San Francisco, CA, USA, June 2017, vol. 3, pp. 1991-1998.

[5] [5].S.V. Syromukov, et al "NG-24 Neutron Generator for Nuclear Medicine and Thermonuclear Research," Atomic Energy, vol. 119, pp. 58-60, October 2015

[6] IBR-2 Reactor Parameters. Available: http://flnph.jinr.ru/en/facilities/ibr-2/parameters. Accessed on: 08 12, 2021.

[7] Yu.G. Dragunov, et al "Modernization of the IBR-2 pulsed research reactor,” Atomic Energy, vol. 113, pp. 29-38, November 2012. 